\title{
Trans-oral Trans-Sheath Forceps Biopsy For Patients With Severe Esophageal Obstruction Under Fluoroscopy
}

\section{Dechao Jiao}

The First Affiliated Hospital of Zhengzhou University

Kaihao Xu

The First Affiliated Hospital of Zhengzhou University

Yiming Liu

The First Affiliated Hospital of Zhengzhou University

\section{Zongming $\mathrm{Li}$}

The First Affiliated Hospital of Zhengzhou University

\section{Yanli Wang}

The First Affiliated Hospital of Zhengzhou University

\section{Jianzhuang Ren}

The First Affiliated Hospital of Zhengzhou University

Xinwei Han ( $\sim 13592583911 @ 163 . c o m)$

The First Affiliated Hospital of Zhengzhou University

\section{Research article}

Keywords: forceps biopsy, esophageal obstruction, fluoroscopy

Posted Date: November 17th, 2021

DOI: https://doi.org/10.21203/rs.3.rs-1071793/v1

License: (9) This work is licensed under a Creative Commons Attribution 4.0 International License. Read Full License 


\section{Abstract}

Background: To investigate the safety and effectiveness of trans-oral trans-sheath forceps biopsy (TTFB) for patients with severe esophageal obstruction under fluoroscopy.

Methods: From November 2016 to November 2019, 35 patients with level $\rrbracket$ or $\rrbracket$ dysphagia and a Karnofsky score of less than 60 were enrolled to undergo TTFB and esophageal nutrition tube insertion or stenting simultaneously. Data on diagnostic performance, early complications, and radiation dose were collected, and Karnofsky scores before and after the procedures were compared.

Results: The technical success of TTFB was $100 \%$. The sensitivity, specificity and accuracy were $92.3 \%$ (24/26), $77.8 \%$ (7/9), and 88.6\% (31/35), respectively. Complications occurred in two cases (5.7\%). The mean procedure duration and irradiation dose were $23.2 \mathrm{~min}$ and $7.2 \mathrm{mSv}$, respectively. The Karnofsky scores significantly increased after $2-4$ weeks $(t=-8.9, P<0.01)$.

Conclusions: TTFB is a safe and effective method for patients with severe esophageal obstruction under fluoroscopy, especially in those who cannot undergo or refuse endoscopy.

\section{Background}

Esophageal cancer is a common malignant tumor with a poor prognosis; China accounts for more than $50 \%$ of esophageal cancers worldwide [1, 2]. The early symptoms of esophageal cancer are not obvious, and most patients are at the middle or late stage when diagnosed. Endoscopy can afford visualization of the esophageal surface, the extent of tumor involvement, ulcer, and diverticulum, and can with the help of intracavitary ultrasound or fluorescence technology facilitate the gathering of samples for a clear diagnosis [3, 4]. Although endoscopic biopsy (EB) has been widely used to obtain samples from the stenosed part, it is not feasible in some patients for various reasons such as patients' refusal of endoscopic treatment, inability to tolerate anesthesia, and poor constitution, and presence of severe stricture, especially in patients with level 3 or 4 dysphagia and a Karnofsky score less than 60 points. In this study, we introduced a trans-oral trans-sheath forceps biopsy (TTFB) method to obtain esophageal tissue from the occlusion site under local anesthesia.

\section{Methods}

\subsection{Patients}

This retrospective single center study was approved by our ethics review committee. From November 2016 to November 2019, 35 patients (20 men and 15 women, mean age: $74.3 \pm 5.6$ years) with level 3 or 4 dysphagia and a Karnofsky score less than 60 were enrolled to undergo TTFB and nutrition tube insertion simultaneously. The reasons for choosing TTFB were poor condition $(n=26)$, refusal to undergo endoscopy $(n=7)$, and inability to tolerate anesthesia $(n=2)$. The mean stenosis length and maximum tumor diameter were 48.7 and 22.3 mm, respectively. More study details are listed in Table 1. 
Table 1

Study data

\begin{tabular}{|c|c|}
\hline Date & Value (range or \%) \\
\hline Total patients & 35 \\
\hline Mean age, years(range) & $74.3 \pm 5.6(63-85)$ \\
\hline Sex(male/female) & $20 / 15$ \\
\hline Location(upper/middle/lower) & $9 / 18 / 8$ \\
\hline Stenosis length (mm) & $48.7 \pm 14.5(21-80)$ \\
\hline Maximum tumor diameter(mm) & $22.3 \pm 5.4(13-37)$ \\
\hline 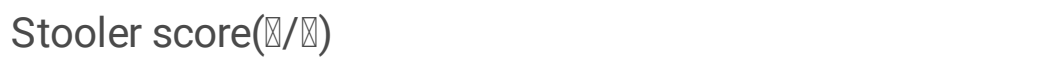 & $16 / 19$ \\
\hline \multicolumn{2}{|l|}{ Previous treatment (\%) } \\
\hline No & 19(28.6) \\
\hline Stenting & $8(22.9)$ \\
\hline Irradiation & $7(20)$ \\
\hline Chemotherapy & $4(11.4)$ \\
\hline \multicolumn{2}{|l|}{ The reasons for forceps biopsies } \\
\hline Poor conditions & $26(74.2)$ \\
\hline Refusal of endoscopy & $7(20)$ \\
\hline Anesthesia problems & $2(5.8)$ \\
\hline Mean number of biopsies(range) & $5.2(2-8)$ \\
\hline Mean procedure duration(min) & $21.9 \pm 4.5(15-32)$ \\
\hline Mean irradiation dose & $7.2 \mathrm{mSv}(2.4-11.9)$ \\
\hline Balloon dilatation assistance (N/balloon diameter, $\mathrm{mm}$ ) & $7 / 8$ \\
\hline \multicolumn{2}{|l|}{ Karnofsky score } \\
\hline Before & $51.1 \pm 7.6(40-60)$ \\
\hline After & $63.1 \pm 9.0(40-80)$ \\
\hline Technical success rate $(\%)$ & 100 \\
\hline Sensitivity (\%) & $92.3(24 / 26)$ \\
\hline Specificity (\%) & $77.8(7 / 9)$ \\
\hline Accuracy (\%) & $88.6(31 / 35)$ \\
\hline
\end{tabular}




\begin{tabular}{|l|l|}
\hline \multicolumn{1}{c|}{ Date } & Value (range or \%) \\
\hline Minor bleeding (\%) & 2 \\
\hline Final pathology & 28 \\
\hline Esophageal carcinoma & 7 \\
\hline Granulation tissue &
\end{tabular}

For the procedure, the patients were supine on a digital subtraction angiography (DSA) examination bed. The patients' oxygen inhalation was monitored and electrocardiograms were obtained. The esophageal tube was inserted under local anesthesia (oral) with $5 \mathrm{ml}$ of $2 \%$ lidocaine. Using a 0.035 -inch guidewire (Cook Bloomington IN, USA), the catheter was manipulated to the stomach through the esophageal stenosis; another angiography was performed through the catheter to measure the esophagus stricture or occlusion. The upper segment of the occluded part was dilated and the stricture length was recorded. Thereafter, the catheter was again manipulated toward the stomach and the guidewire was replaced with a super-stiff guidewire, along which a $10 \mathrm{~F}$ long sheath $(80 \mathrm{~cm}$ in length, Cook Bloomington IN, USA) was passed. The sheath end was pressed toward the narrow esophageal part. Then, the biopsy forceps was introduced into the sheath, opened, and pushed forward by 5-10 mm, after which the forceps was tightened to obtain the tissue sample (Fig. 1 and Fig. 2). After withdrawing the biopsy forceps, the diseased tissue was fixed in $10 \%$ formaldehyde and sent for histological examination. After 2-8 repetitions of theaforementioned process, at least two pieces of the tissue were successfully clamped. The specimens obtained were immediately evaluated by a pathologist, after which the operation was finished and the device was withdrawn. Then, the guidewire and catheter were manipulated with each other through nose-esophagus until they finally reached the stomach. The catheter was replaced with a nutritional tube, which was attached to the side of the face to hold it in place.

\subsection{Definition}

The final results were obtained by reviewing the pathological results, final surgical operation findings, or follow-up medical images. The evaluation standards were as follows: (1) pathology results for the final surgical resection, (2) pathological results were accepted as corrected diagnosis such as malignant or specific benign results such granulation hyperplasia, hamartoma or tuberculosis; (3) for negative for malignancy, fibrous tissue, and inflammation results, follow-up imaging or endoscopy were used to decide the true or false results within 3 months.

\subsection{Statistical analysis}

All data were recorded and analyzed using SPSS software (version 17.0, USA). Karnofsky scores were compared using a paired t-test, and significance was set at a P-value of 0.05 .

\section{Results}




\subsection{General records}

The mean patient age was 74.3 years. The tumor was located in the upper, middle, and lower parts in nine, 19, and eight cases, respectively. Stenosis length and tumor diameter were $48.7 \mathrm{~mm}$ (range: 21-80 $\mathrm{mm}$ ) and $22.3 \mathrm{~mm}$ (range: $13-37 \mathrm{~mm}$ ), respectively. The technical success of TTFB was $100 \%$. The mean procedure duration was $23.2 \mathrm{~min}$, resulting in a mean exposure dose of $7.2 \mathrm{mSv}$. The nutrition tube was used in 27 cases (77.1\%), and an esophageal stent was inserted after TTFB in eight cases $(22.9 \%)$. The pre- and post-procedure Karnofsky scores were $51.1 \pm 7.6(40-60)$ and $63.1 \pm 9.0$ (40-80), respectively, with there being a significant difference between the scores $(t=-8.9, P<0.01)$. More detailed information is provided in Table 1.

\subsection{Pathological results and complications}

The mean number of biopsies was 5.2, and the number of samples from all patients was sufficient for diagnosis under the pathologist's supervision. Diagnoses were missed in four patients (11.4\%), of whom three showed epithelial dysplasia while the fourth patient showed a large amount of fibrous connective tissue that was suspected to be part of heterogeneous cells. Three of these patients received nasal feeding, and their physical status improved 3 weeks later, and the surgeries in these patients indicated constrictive esophageal cancer. The fourth patient was followed up and showed aggravated hyperplasia at the ends of the stent. After an improvement in the patient's general health within one month, biopsy under gastroscopy was repeated, and it indicated squamous cell carcinoma. Thus, the sensitivity, specificity, and accuracy rate were $92.3 \%(24 / 26), 77.8 \%$ (7/9), and $88.6 \%(31 / 35)$. Complications occurred in $2(5.7 \%)$ cases, with both patients showing some hematemesis ( $<5 \mathrm{~mL})$, and all patients' symptoms disappeared after injecting epinephrine $(1 \mathrm{mg})$ through the sheath without further intervention. No esophageal perforation or massive hemorrhage was found.

\section{Discussion}

Digestive endoscopy has significantly increased the rates of early detection of esophageal cancer or precancerous mucosal lesions [5]. Gastroscopy can allow direct observation of the lesions of the esophageal mucosa, especially for swelling and ulcerative lesions. EB can be performed simultaneously, which is generally accepted as one of the best ways to obtain the sample from esophageal cancer pretreatment, and the accuracy of endoscopic forceps biopsy was $60 \%-95 \%$ in previous literature [6-8]. However, some patients cannot undergo EB due to anesthesia-related risks, age-related factors, severe occlusion, refusal, and other reasons, and appropriate diagnosis in these patients remains a clinical difficult problem. The most ideal condition in forceps biopsy is to obtain the least amount of biopsy tissue from the most typical part to reflect the overall nature of the lesion [9]. For patients with severe esophageal stenosis, the tumor information is hidden across the stenosis area and the approach used for obtaining pathological tissue out of the stenosis area is very important. In the past, Li TF et al have reported that TTFB can be used to diagnose malignant obstructive jaundice; thus, it can hypothetically be used to diagnose esophageal strictures [10]. In addition, some patients don't even have water for several 
weeks, the general condition is very poor, nutritional support is still the most basic and important treatment for these patients. We can complete forceps biopsy and provide a nutrition tube or esophageal stent placement simultaneously under local anesthesia to improve the efficiency of diagnosis and treatment.

In this pilot study, 35 patients underwent TTFB for esophageal stenosis. The technical success rate was $100 \%$ and sufficient histologic specimens were obtained. The final pathological accuracy rate was $88.6 \%$, which was satisfactory, and was within the accuracy range of previous reports [6-8]. The stenoses were relatively narrow, and biopsy forceps were manipulated closely to the beginning of the lesion at the head of the sheath so as to obtain samples out of lesion sidewalls. In terms of complications, two cases showed minor self-limiting hemorrhage, which is reasonable in comparison with that associated with the use of clamp forceps under endoscopy, and the safety was satisfactory [11]. However, TTFB has certain disadvantages such as unclear visualization of the mucosal surface, outline, and scope of the lesion and the incompatibility with narrow-band imaging technology. In addition, it is difficult to sample deeper submucosal lesions with such forceps. Diagnoses were missed in four patients, who were finally confirmed as showing cancer. This is still a practical problem, and a non-specific outcome in this examination cannot rule out the presence of malignant tumors, necessitating further evaluations. The location, method, quantity, forceps times, and specimen preparation may affect the assessment [12]. As for irradiation dose, $7.2 \mathrm{mSv}$ is similar to the radiation dose in a standard CT chest scan (7 mSv) [13], which is reasonable in our opinion, although the irradiation dose can be further reduced by using a small field of view and improving work efficiency.

In order to obtain more samples in patients with esophageal stenosis, our technical experience is as follows: (1) The long sheath must be introduced along a super-stiff guidewire and should be as close to the stenosis as possible. (2) After introducing the forceps through the outer sheath, the forceps can be opened and then pushed forward 5-10 mm into the stenosis so as to obtain more samples. (3) If the stenosis is very severe, forceps biopsy should be performed after dilatation with a small balloon dilator (diameter, 6-8 mm). Larger balloons (diameter $\geq 10 \mathrm{~mm}$ ) are not recommended. When no stenosis indicates no support platform at the head of the sheath, it is easy to slide to the distal end of the stenosis. In such cases, the opening biopsy forceps cannot touch the sidewall of the lesion, and the forceps cannot get close to the diseased wall leading to sampling failure, or a large balloon can compress the esophageal cells leading to denaturation or necrosis. (4) Multiple biopsies may be required because the obstructive disease is actually caused by the lesion itself and the inflammatory edema around it. The biopsy forceps must be used in a very limited part of the middle of the lesion while obtaining the lesion tissue; otherwise, it may also collect inflammatory tissue. Therefore, it is necessary to forceps the stenosis in different parts and directions, but multiple biopsies do not imply the use of a higher number of clamps, which is also important to reduce complications while diagnosing diseases. (5) The diagnosis of tissue sections should be performed promptly by the pathology department, and the tissue dehydration time should not be too long; otherwise, the specimen will undergo compression, degeneration, and necrosis. 
However, this study had its own limitations, such as the small number of patients, biased selection, and the absence of a prospective design and a control group.

In conclusion, TTFB is a safe and effective method for patients with severe esophageal obstruction under fluoroscopy, especially those who cannot undergo EB due to anesthesia-related risks, age-related factors, severe occlusion, or refusal to undergo endoscopy.

\section{Abbreviations}

TTFB: trans-oral trans-sheath forceps biopsy; EB: endoscopic biopsy.

\section{Declarations}

\section{Authors' contributions}

Dechao Jiao: Formal analysis; Methodology; Roles/Writing-original draft; Writing-review and editing. Kaihao Xu: Data curation. Yiming Liu: Resources. Zongming Li: Conceptualisa-tion. Yanli Wang: Supervision. Jianzhuang Ren \& Xinwei Han: Writing-review. All authors read and approved the final manuscript.

\section{Funding}

This work was supported by none of funding.

\section{Availability of data and materials}

The clinical data were obtained from the interventional department of the First Affiliated Hospital of Zhengzhou University. The data used to support the findings of this study are available from the corresponding author upon request.

\section{Ethics approval and consent to participate}

All procedures performed in the studies involving human participants were in accordance with the ethical standards of the institutional and/or national research committee and with the 1964 Helsinki Declaration and its later amendments or comparable ethical standards.

\section{Consent for publication}

Subject to acceptance, the authors will sign an exclusive licence to publish.

\section{Competing interests}

There are no ethical issues or conflicts of interest. 


\section{References}

1. M MH, Ivanovski I, Spartalis E, Chrysikos D, Athanasiou A. Diaphragmatic hernia following esophagectomy for esophageal cancer: A systematic review. Journal of BUON: official journal of the Balkan Union of Oncology 2019;24(5):1793-800.

2. Li ZM, Lu HB, Ren KW, Han XW, Wu G, Jiao DC. Thoracic stomach-right main bronchus fistula treated with dual Y-shaped covered airway stents. Clin Radiol 2017;72(6):517.e1-.e6.

3. Ebi M, Sakamoto K, Inoue S, Ozeki T, Kimura M, Kondo R, et al. Esophageal Leiomyosarcoma Diagnosed by Endoscopic Ultrasound-guided Fine-needle Aspiration Biopsy and Cured with Surgical Resection. Intern Med 2019;58(17):2479-83.

4. Abdullah HM, Ullah W, Abdallah M, Khan U, Hurairah A, Atiq M. Clinical presentations, management, and outcomes of acute esophageal necrosis: a systemic review. Expert Rev Gastroenterol Hepatol 2019;13(5):507-14.

5. Liu M, Liu Z, Liu F, Guo C, Xu R, Li F, et al. Absence of lodine Staining Associates With Progression of Esophageal Lesions in a Prospective Endoscopic Surveillance Study in China. Clin Gastroenterol Hepatol 2020;18(7):1626-35. e7.

6. Furneri G, Klausnitzer R, Haycock L, Ihara Z. Economic value of narrow-band imaging versus white light endoscopy for the diagnosis and surveillance of Barrett's esophagus: Cost-consequence model. PLoS One 2019;14(3):e0212916.

7. Walther C, Jeremiasen M, Rissler P, Johansson JL, Larsson MS, Walther BS. A New Method for Endoscopic Sampling of Submucosal Tissue in the Gastrointestinal Tract: A Comparison of the Biopsy Forceps and a New Drill Instrument. Surg Innov 2016;23(6):572-80.

8. Lee DS, Ahn YC, Eom DW, Lee SJ. Primary esophageal mucosa-associated lymphoid tissue lymphoma diagnosed by using stacked forceps biopsy. Dis Esophagus 2016;29(7):887-90.

9. Falk GW, Rice TW, Goldblum JR, Richter JE. Jumbo biopsy forceps protocol still misses unsuspected cancer in Barrett's esophagus with high-grade dysplasia. Gastrointestinal endoscopy 1999;49(2):170-6.

10. Li TF, Ren KW, Han XW, Li WC, Ren JL, Jiao DC, et al. Percutaneous transhepatic cholangiobiopsy to determine the pathological cause of anastomotic stenosis after cholangiojejunostomy for malignant obstructive jaundice. Clin Radiol 2014;69(1):13-7.

11. Muto M, Minashi K, Yano T, Saito Y, Oda I, Nonaka S, et al. Early detection of superficial squamous cell carcinoma in the head and neck region and esophagus by narrow band imaging: a multicenter randomized controlled trial. J Clin Oncol 2010;28(9):1566-72.

12. di Pietro M, Canto MI, Fitzgerald RC. Endoscopic Management of Early Adenocarcinoma and Squamous Cell Carcinoma of the Esophagus: Screening, Diagnosis, and Therapy. Gastroenterology 2018;154(2):421-36.

13. Mettler FA, Jr., Huda W, Yoshizumi TT, Mahesh M. Effective doses in radiology and diagnostic nuclear medicine: a catalog. Radiology 2008;248(1):254-63 


\section{Figures}
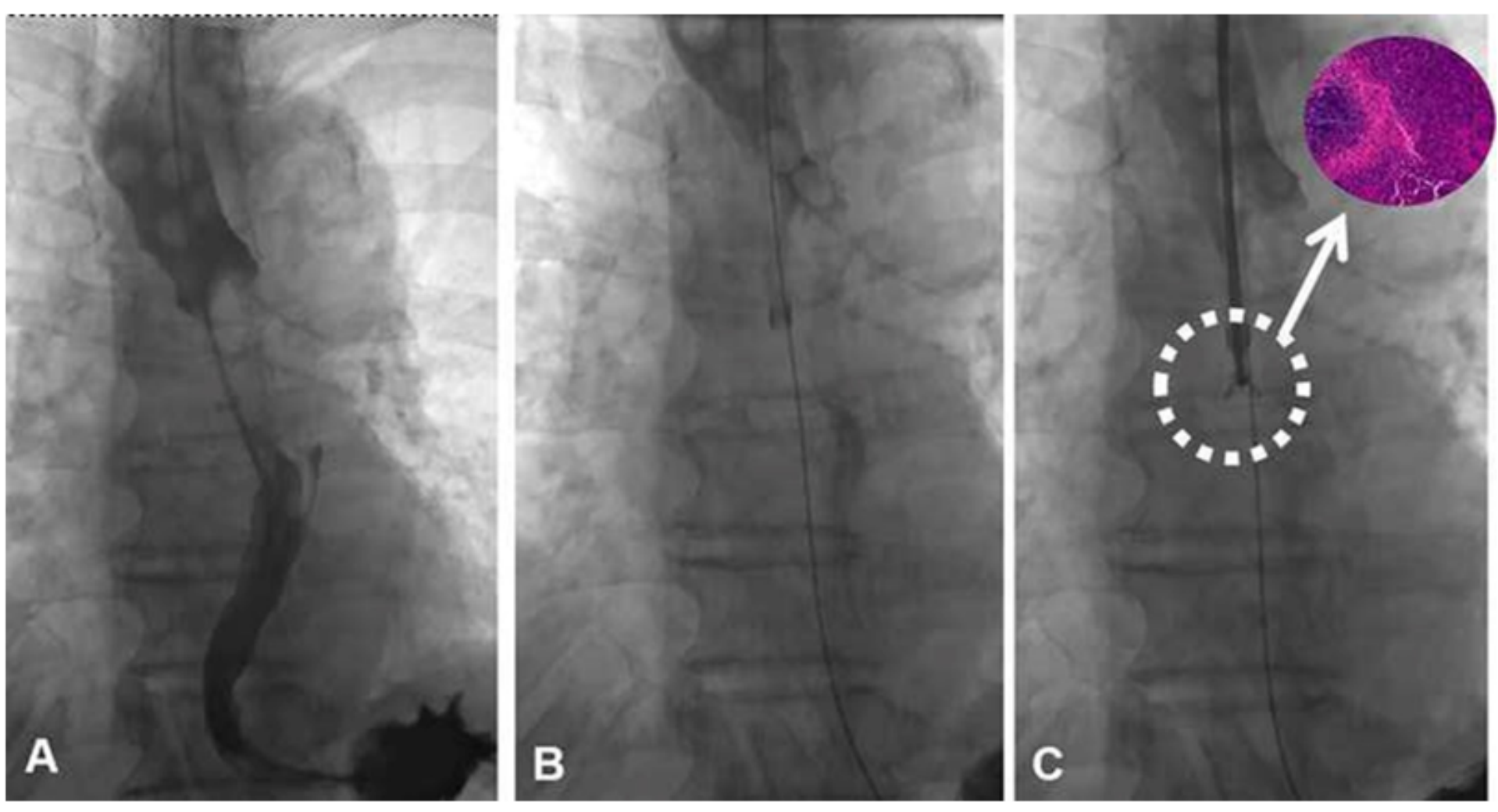

\section{Figure 1}

76-year-old male presented with level 3 dysphagia. A: Catheter angiography demonstrated esophageal interruption and filling defect. B: The sheath was introduced along a super-stiff guidewire toward the stenosis part of the lesion. C: The biopsy forceps was introduced through the sheath to clamp the samples, and squamous cell carcinoma was confirmed by pathology. 

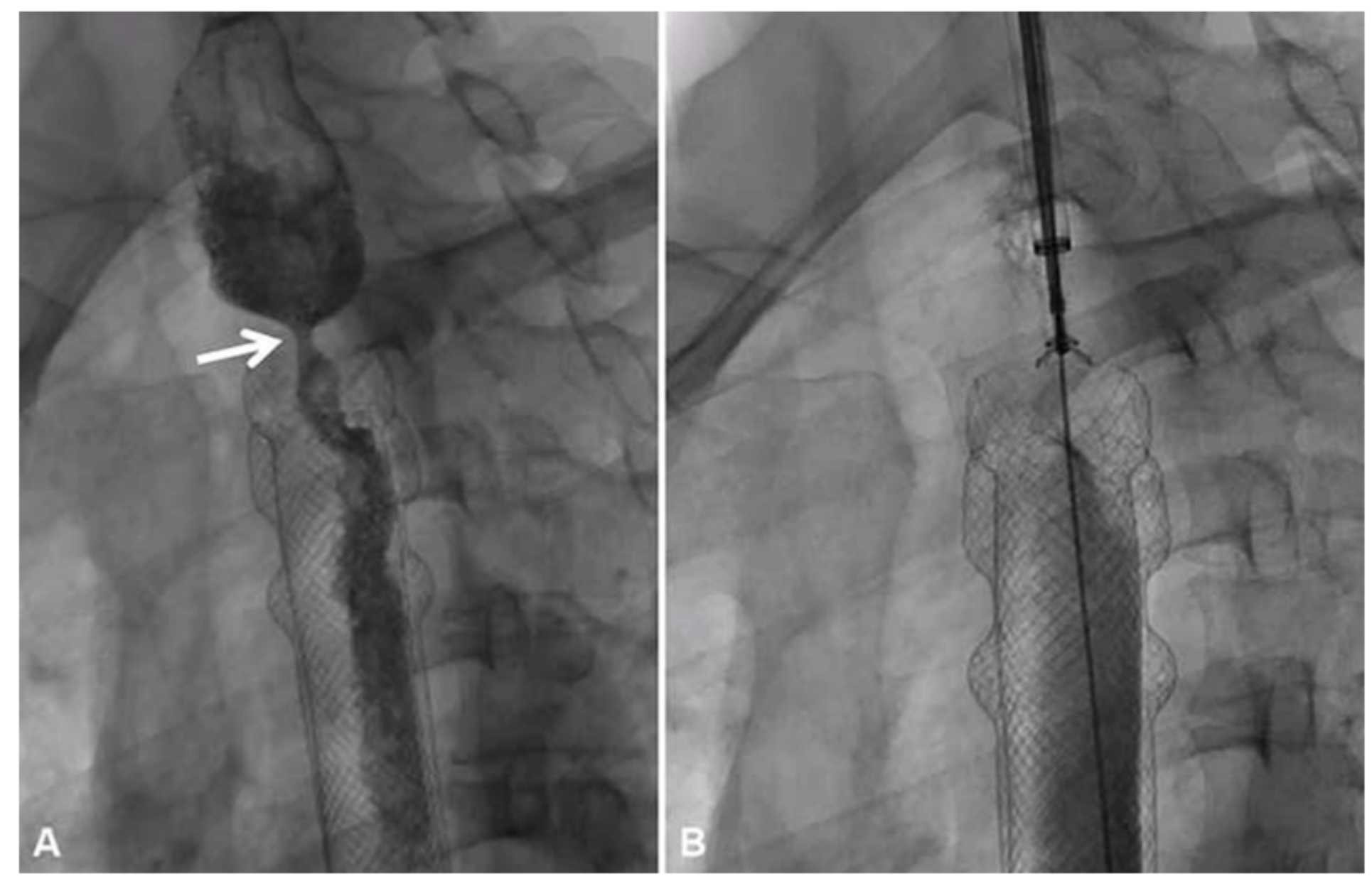

\section{Figure 2}

57-year-old male presented with level 3 dysphagia. A: Catheter angiography demonstrated esophageal stenosis in the upper part of the esophageal stent. B: The biopsy forceps was introduced through the sheath to clamp the samples, and granulation hyperplasia was confirmed by pathology. 